\title{
A Smart Grid-Based Home Energy Aware System
}

\author{
Shang Yuan Chen ${ }^{*}$ and Zhen Lun Hu \\ School of Architecture, Feng Chia University, No. 100, Wenhwa Rd., Seatwen, Taichung 40724, Taiwan, R.O.C.
}

(Received April 6, 2017; accepted July 27, 2017)

Keywords: smart grid, energy management, context awareness, intuitive design, energy efficiency

In this study, we propose the use of a home energy aware system (HEAS) and an intuitive user interface to help household occupants to be aware of the states of different types of power generation and unconsciously improve their electrical use behavior, and thereby achieve the goal of reducing mains electricity consumption. Because of the application of a smart grid and a smart meter infrastructure, smart homes can use various energy management approaches, such as alternative energy, energy storage, and mains electricity. This system employs the National Instruments data acquisition (NI DAQ) energy data retrieval system and LabVIEW software as core programming technologies, providing four power usage reminders: alternative energy use reminder, stored energy power use reminder, insufficient stored energy warning, and mains electricity use reminder.

\section{Introduction}

A home energy aware system (HEAS) can be defined as an awareness system under a "smart grid" and a smart meter infrastructure aimed at household members, and established under the level of the home energy management system (HEMS). A HEAS has the goal of providing home users with clearly and easily understood reminders of power supply or usage to encourage users to unconsciously improve their power use habits. A HEAS overlaps with the scope of a smart grid and energy management, and encompasses research dimensions including industrial analysis, energy systems and technologies, energy management systems and technologies, and HEMSs and technologies.

\section{Literature Review and Case Studies}

A smart grid has been defined as the use of automatic monitoring and control technologies, in conjunction with information and communications technology, to assist effective grid operational planning and strengthen user participation, and combines an electric power system with information and communications technology and services to achieve the goals of energy conservation and reduction in carbon emission. ${ }^{(1,2)}$ In a smart grid facility, on the demand side, information expressing the status of various power sources can be actively provided to consumers via an energy management system; thus, the development of this system represents an important new challenge. ${ }^{(3)}$

*Corresponding author: e-mail: shangyuanc@gmail.com

http://dx.doi.org/10.18494/SAM.2017.1660 
According to Yang (2008), building energy management system (BEMS) applications have been developed since 1955. ${ }^{(4)}$ Nevertheless, to date, from both academic and practical viewpoints, the development of intelligent HEMSs in a smart grid infrastructure is still at an extremely early stage. Researchers have proposed various mathematical models and algorithms to describe the differences in the management of conventional centralized and distributed power supply systems. MohsenianRad et al. (2010) suggested that the distributed power in the form of smart grids is an optimal energy conservation strategy and enables the achievement of Nash equilibrium. ${ }^{(5)}$ Khanna (2012) believes that the incorporation of unpredictable renewable energy in power grids will prompt the development of wide-area control algorithms. ${ }^{(6)}$ Apart from this, other researchers have explored the use of information and communication technologies, such as wireless transmission equipment in power monitoring and control systems. ${ }^{(7)}$

A HEMS is an energy management system aimed at household users and includes power supply, energy consumption, energy management, energy storage, and energy cycle management mechanisms. Wang (2013) explained a HEMS as employing various information and communications technologies to sense and collect information on energy usage by household occupants, and relying on the analysis of the power use behavior to provide optimized energy conservation measures to guide home energy management. The realization of such a system will require the development of visualization technology, automation and control technology, and analytical functions. ${ }^{(8)}$ Based on mains supply as main power source tradition, the majority of research studies on HEMSs still focus on the monitoring and/or management of domestic appliance energy use, which aims to schedule the operation of appliances. ${ }^{(9-11)}$

At the level of practical applications, according to NRGExpert (2011), HEMSs are still at a very early stage in terms of development and applications. ${ }^{(12)}$ Because the distributed power system concept and technologies such as smart grids, smart meters, and smart appliances are still far from ubiquitous, most consumers naturally do not know how to use the tools. As a result, for example, the Google Power Meter product was discontinued after two years of promotion. Table 1 shows four existing, online home energy monitoring and control tools.

The foregoing review of the literature and case analysis reveals that online energy monitoring and management tools rely on smart meters as power information sensing elements, and employ wireless communications technology, computer processing capacity, and control technology to monitor and analyze power usage (Fig. 1). However, existing HEMSs emphasize user-end power consumption and can monitor and control domestic appliance energy use. Nevertheless, there is as yet little understanding of distributed power supply models and application methods in a smart grid/smart meter infrastructure. To encourage consumers to use various forms of distributed power generation (including alternative energy and energy storage) and reduce their dependence on mains electricity, system and interface developments must place particular emphasis on the provision of power supply information. As a consequence, on the demand side, the development of a HEMS that can actively provide information on various power sources to users, and can give users easily understood, useful information, is a key new challenge.

\section{Theory and Method}

To encourage consumers' use of various forms of distributed power generation (including alternative energy and energy storage), and reduce their consumption of mains electricity, this study advocates the development of a HEAS and a user interface possessing the features of context 
Table 1

(Color online) Overview of home energy monitoring tools.

\begin{tabular}{|c|c|c|c|c|}
\hline Item & $\begin{array}{l}\text { Google Power Meter } \\
\text { monitoring interface }{ }^{(13)}\end{array}$ & $\begin{array}{l}\text { Microsoft Hohm } \\
\text { monitoring interface } \\
\text { (14) }\end{array}$ & $\begin{array}{l}\text { San Der Technology's } \\
\text { ASENs-910 series }\end{array}$ & In-Snergy, iFamily ${ }^{(16)}$ \\
\hline Image & 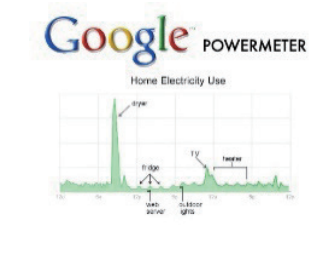 & 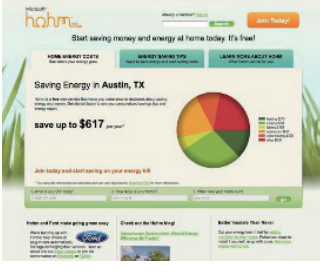 & 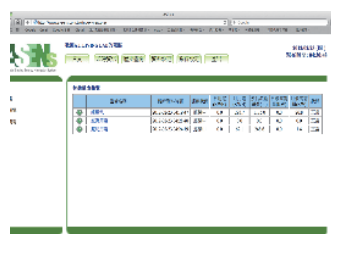 & 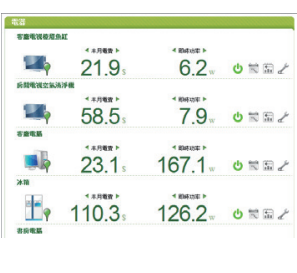 \\
\hline Available & 2009/02-2011/09 & June 24, 2009-2012 & Unclear date-until now & 2013-until now \\
\hline
\end{tabular}

Explanation Consumers can use smart meters and energy devices to observe the real-time power use of household equipment via the Internet.

Restrictions The interface displays the power usage of household equipment. However, consumers cannot easily distinguish good from bad power consumption because of the lack of necessary usage analysis and comparisons.
Microsoft Hohm has functions similar to those of the Google Power Meter, but is also able to compare and analyze the power consumption of households with similar sizes and areas, which helps in providing power-saving recommendations.

The interface emphasizes the consumer power use behavior, but fails to express the distribution and usage of various power sources on the smart grid. It is designed still based on mains supply tradition.
Using Zigbee wireless transmission technology, smart meters can view current power information in real time via the Internet, and allow users to query historical data and statistics, including daily/ monthly power consumption and maximum demand.

Chiefly uses tables, statistics, and analytical charts to express data, and employs much opaque jargon. As a result, a specialist is required to determine users' power load and use problems on the basis of historical data.

In-Snergy is a cloudbased platform of Smart Green Energy. This application will be able to manage energy consumption, and iFamily is an energy management web interface based on In-Snergy architecture.

The system focuses on the monitoring and/or management of domestic appliance energy use, but lacks the capability to understand the distributions of power generation.

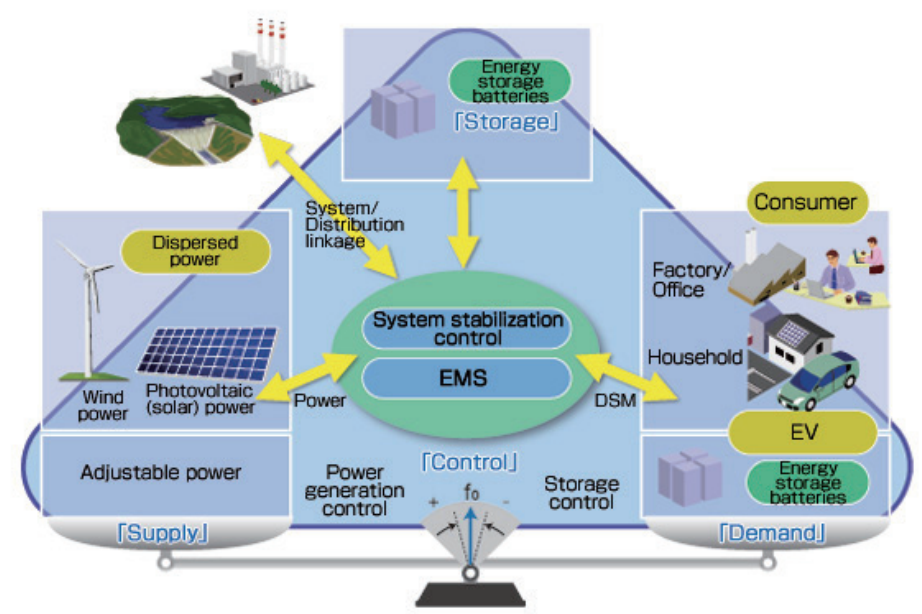

Fig. 1. (Color online) Hitachi's concept for energy management system. ${ }^{(17)}$ 
awareness, intuitive design, and energy efficiency. By ensuring that household occupants can easily understand the power supply status, a HEAS can encourage users to improve their energy usage behaviors. Figure 2 shows the structure of a HEAS with context awareness. In accordance with its mission of energy conservation and cost reduction, this system provides an intuitive interface displaying the real-time status of power supply in a form that is easy for users to see and understand. As a result, the HEAS can encourage household occupants to improve their power use behavior, and thereby improve the power supply situation. Because of this, the use of a HEAS will promote more efficient energy use. In the HEAS, power supply information is sensed and updated at intervals, and the system operates in a cyclic manner. The following is an explanation of the system's aspects of context awareness, intuitive design, and energy efficiency.

\subsection{Context awareness}

"Context" implies information about the current situation, and context awareness implies the use of contextual information. A context-aware system can extract, interpret, and use contextual information and adjust its functions, and the context produced in real time can then be reused. ${ }^{(18-20)}$

\subsection{Intuitive design}

Intuitive design can be achieved only through close teamwork between product or interface designers and developers, who must answer the question "What makes a design seem intuitive?". The most important factor in an intuitive product design is to minimize as much as possible the gap between users' current knowledge or natural tendencies and the target knowledge needed to use the product to perform a task. ${ }^{(21)}$ A product or user interface intended to cause users to change their behaviors can be explained using the Fogg behavior model (FBM), which is in widespread use among designers. According to this model, the product should be able to provide sufficient motivation, have ability that can be implemented, and trigger the implementation of behavior. ${ }^{(22)}$

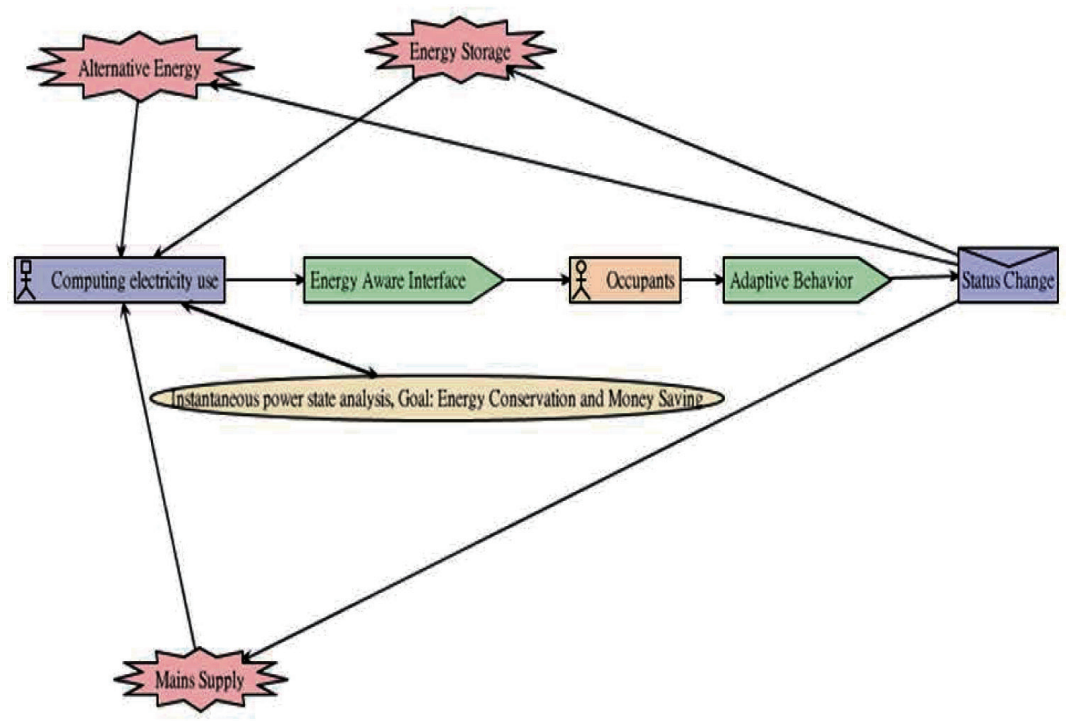

Fig. 2. (Color online) HEAS architecture. 


$$
\text { Behavior }=\text { Motivation } \times \text { Ability } \times \text { Trigger }
$$

\subsection{Energy efficiency}

A HEAS pursues energy efficiency in order to achieve the goal of energy conservation and monetary savings. Patterson (1996) broadly defined energy as ${ }^{(23)}$

$$
\text { Energy efficiency }=\frac{\text { Useful output of a process }}{\text { Energy input of a process }} .
$$

With regard to power sources providing power to the home, in view of the need for environmental protection and economy, alternative energy should take precedence over energy storage, and energy storage should take precedence over mains electricity. Since mains electricity, in particular, requires the calculation of power fees, it is the chief concern of household occupants, and mains electricity usage is closely watched. Because of this, avoidance of the use of costly mains electricity can serve to trigger users' motivation to change their behaviors. In other words, good energy efficiency can be achieved if household occupants are able to obtain relatively large amounts of electricity services while paying relatively low electricity fees during a particular power supply period (such as through the use of alternative energy and energy storage as much as possible).

Furthermore, because power bills are incurred only when mains electricity is used, the formula used to calculate household energy efficiency could be simplified as the ratio of an organization's total power consumption to the mains electricity used during the same period. Therefore, the diagram of "energy efficiency" is as shown in Fig. 3.

\section{Practical Verification}

Taking advantage of the solar panels and two wind turbines already installed on the roof of Feng Chia University's Science and Aeronautical Engineering Building, this project plans to establish an energy use demonstration area in a classroom on the 9th floor. The demonstration area will have a home energy aware interface displaying the supply and use of various types of energy (including

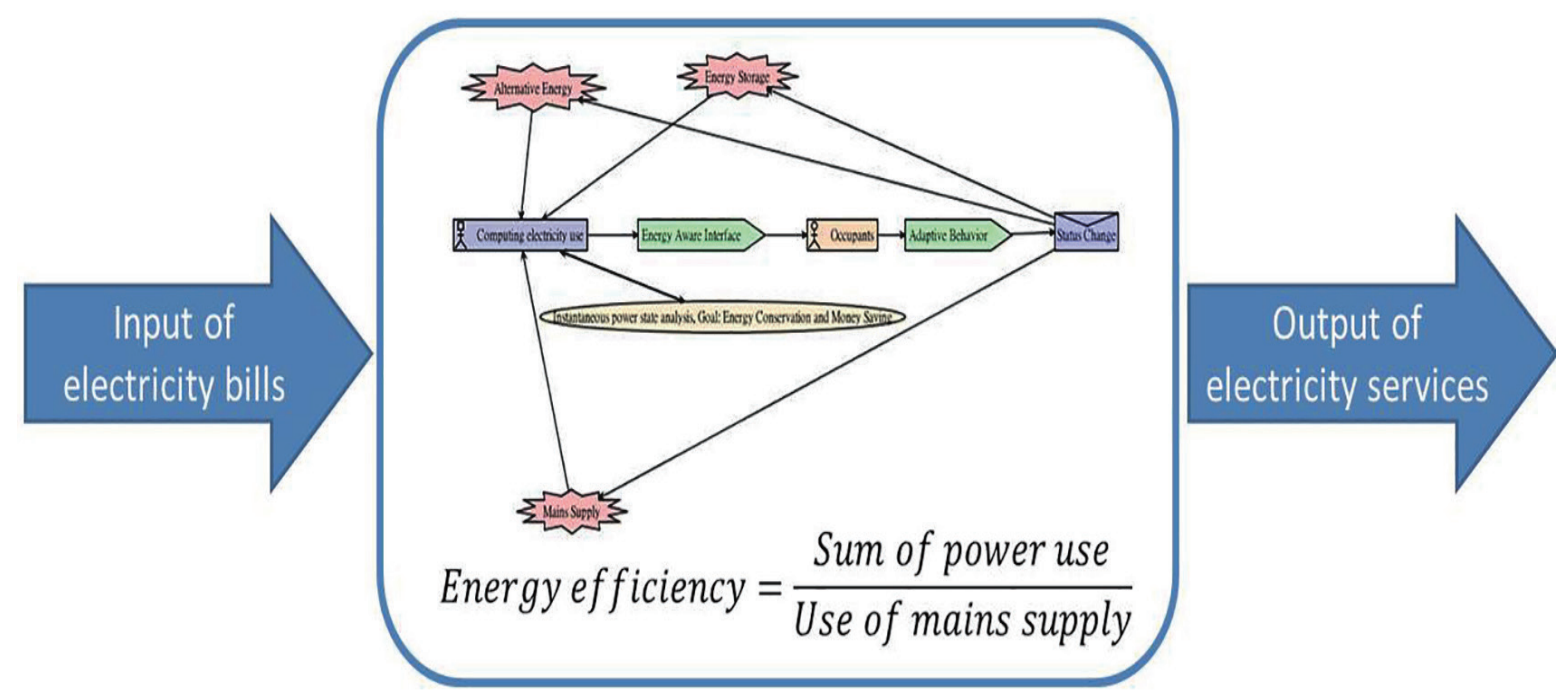

Fig. 3. (Color online) Diagram of energy efficiency. 
alternative energy, power from energy storage batteries, and mains electricity). As shown in Fig. 4, the energy supply data obtained via a data acquisition card is analyzed using a computer, and the interface provides four different power supply information reminder modes to inform users of the current power supply situation. The intuitive user interface encourages occupants to change their power use behaviors in accordance with the state of the power supply, enabling users to pursue energy efficiency and achieve the goal of energy conservation and monetary savings, and shown in Fig. 5 are the exhibition room and the HEAS demonstrated monitor on the wall.

At the technological level, the HEAS and its user interface are constructed using the (1) National Instruments data acquisition (NI DAQ) equipment and (2) LabVIEW interface writing software. The HEAS data acquisition and analysis are performed using the following hardware and software: data acquisition card, enclosures for equipment, computer processor or controller, and interface reading and writing software (Fig. 6).

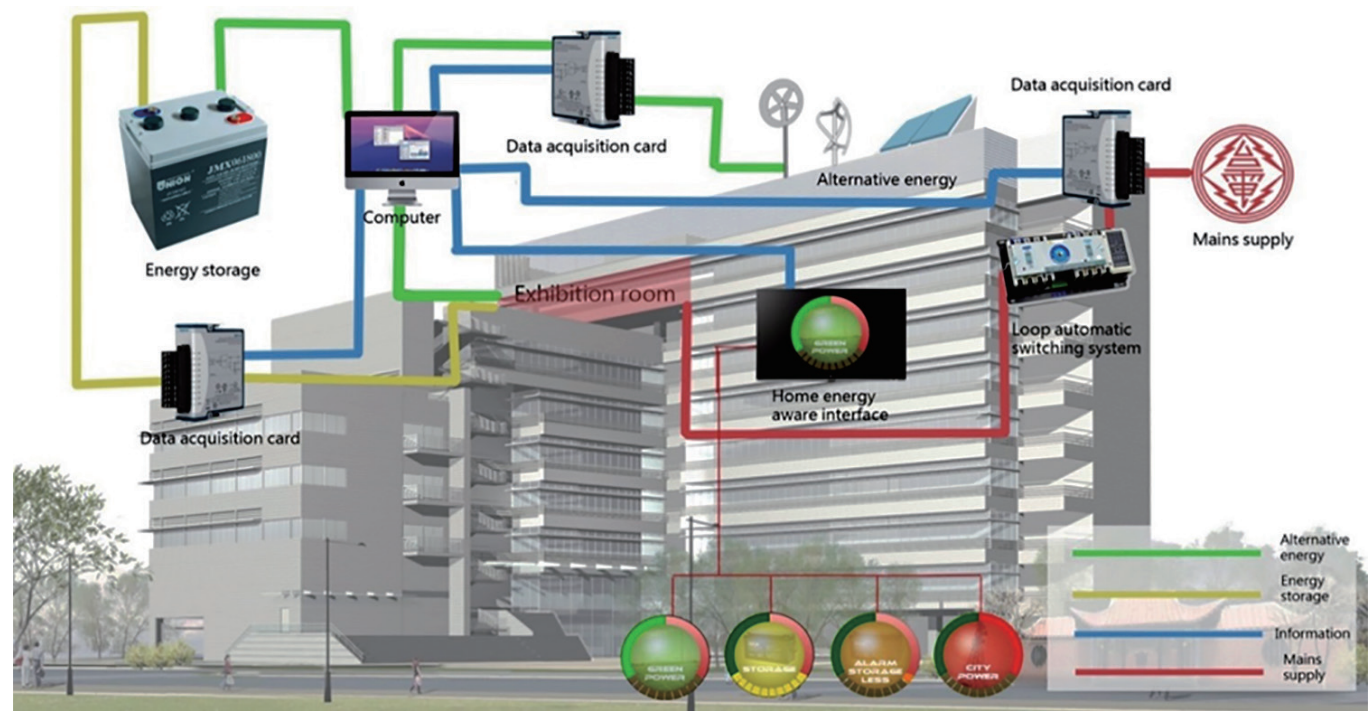

Fig. 4. (Color online) Building a HEAS.

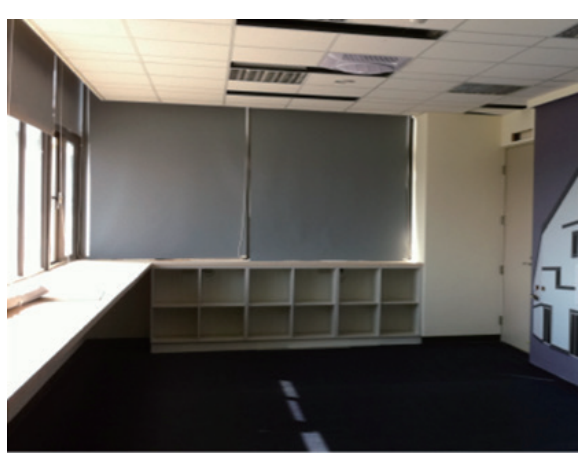

(a)

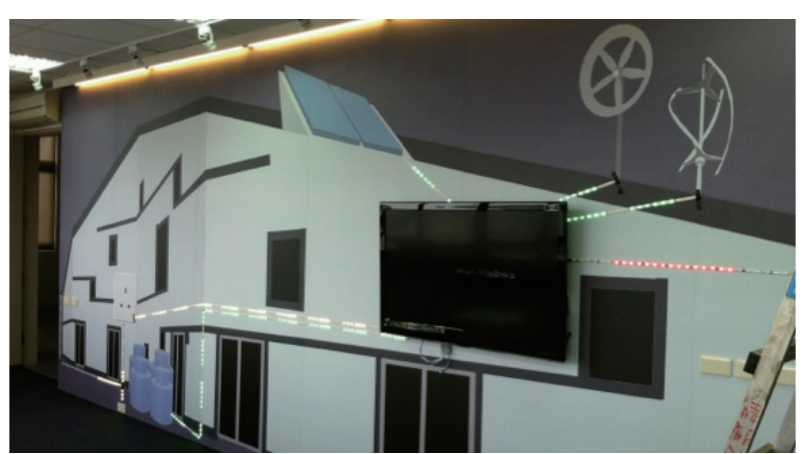

(b)

Fig. 5. (Color online) (a) Exhibition and education classroom and (b) the HEAS demonstrated monitor on the right side. 


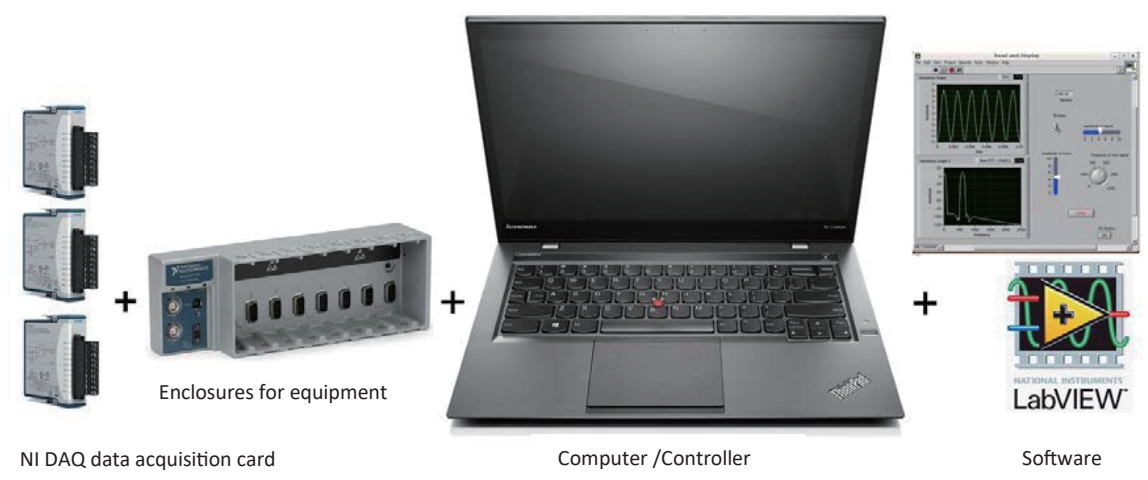

Fig. 6. (Color online) Data acquisition and analysis equipment.

This study uses a NI DAQ card, and this system possesses multiple data monitoring terminals and can simultaneously monitor multiple forms of energy. In this study, the system comprises the power data acquisition end. The data acquisition card has nine input terminals and can read data from nine sources, the signals are passed through a power operational amplifier and a low-noise operational amplifier, and a modem operates on the passing power flow. The data transmitted from the acquisition card currently enters an existing electric meter instrument panel to provide a preliminary information display, and the data is then transmitted to a computer/controller, where data analysis is performed using LabVIEW software (Fig. 7). The home energy aware interface's analysis software was written using the LabVIEW graphic interface writing software, and the syntax contains five parts (Fig. 8):

(1) element controller,

(2) while loop,

(3) local variable display,

(4) for loop, and

(5) user interface.

The display of the image library and current image depends on the "if and then" results of the while and for loops. The following is an example:

Set " $P$ " as the percentage of power from alternative and stored energies.

In steps $1-3$, when $P=100$, there is a sufficient supply of alternative energy, and the EB1 image appears when $\mathrm{Op}$ is 1 , which indicates that power consists of alternative energy.

In steps $4-6$, when $P=0$, there is no power from storage or green energy. At this time, the power consists of mains electricity. The EB13 image appears when Op is 2, indicating that the power is supplied by mains electricity.

In steps 7-9, when $P<20$, the power from energy storage is insufficient to handle the load, the EB2 image appears when the out value is 2, and the power comprises an energy storage low warning indicator.

In steps $10-12$, when $P=99-20$ (else), most power is being supplied from storage. Depending on the percentage, the indicators EB3-EB12 appear when the out value is 11, indicating that power is being supplied from storage, and ten equal intervals are used to display the amount of power in storage.

The display interface uses color to indicate the real-time power supply situation in an intuitive manner. The interface design uses the three colors green, yellow, and red to indicate alternative 


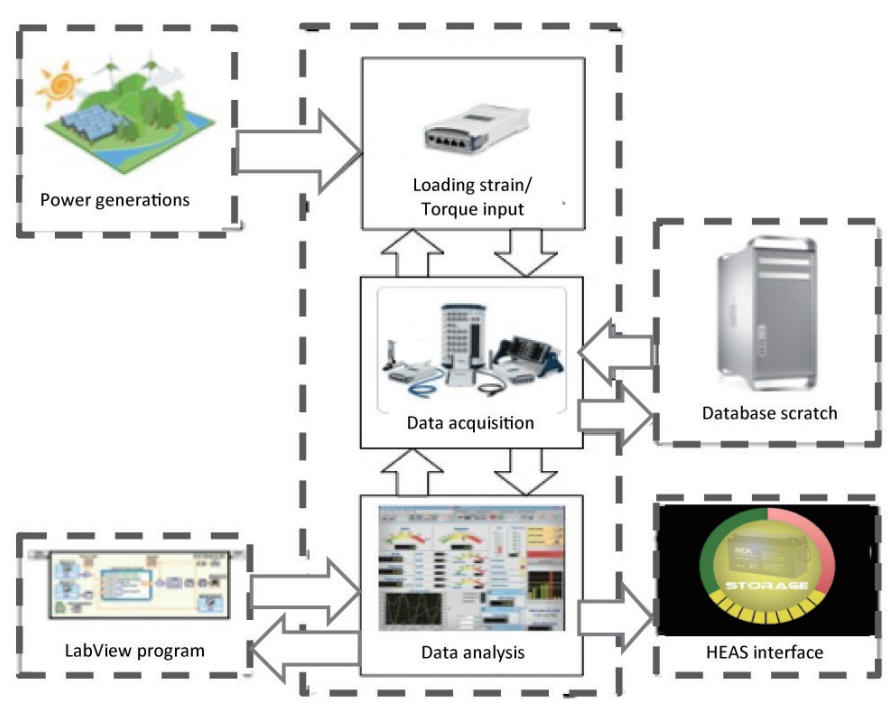

Fig. 7. (Color online) Flow chart of data acquisition and conversion of the HEAS.

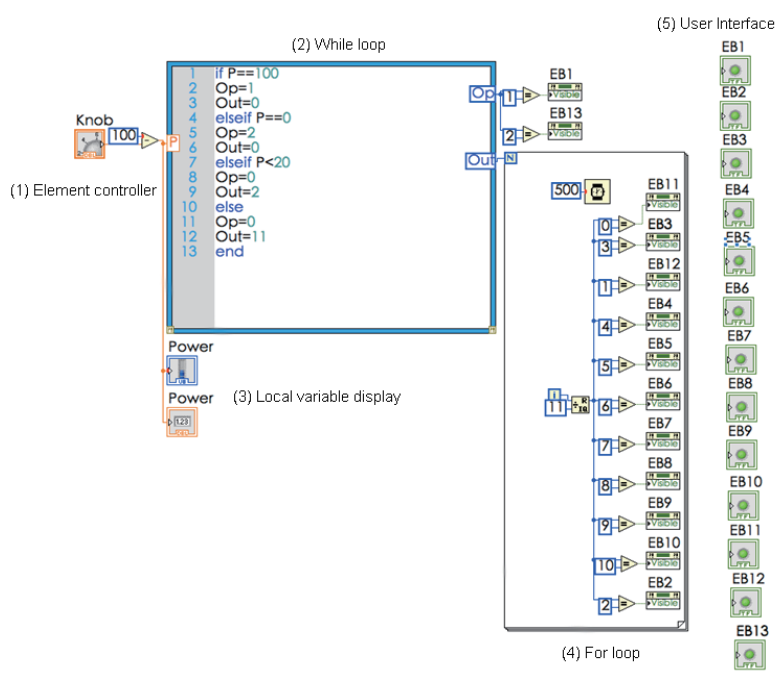

Fig. 8. (Color online) Results of analysis employing a home energy aware interface written using LabVIEW.

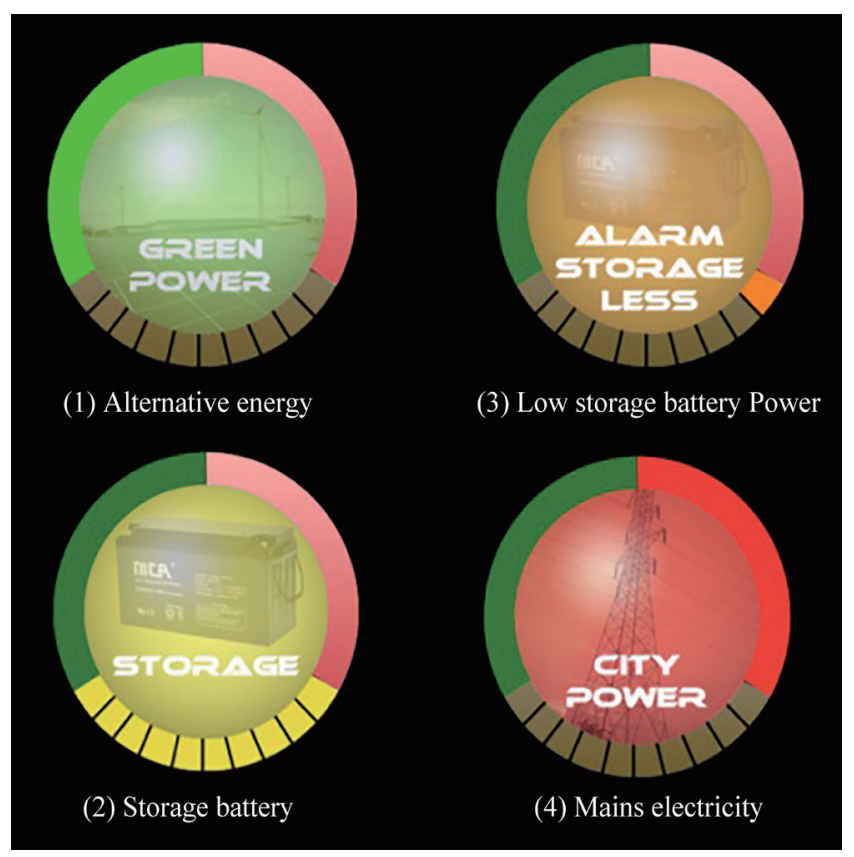

Fig. 9. (Color online) Four different modes in home energy aware interface.

energy, stored energy, and mains electricity, respectively. The results of analysis are expressed in the form of four modes: (1) alternative energy supply mode, (2) energy supply from storage battery mode, (3) low storage battery power warning mode, and (4) mains electricity power supply mode, which notify users of the current state of power supply (Fig. 9). The highly intuitive user interface 
encourages household occupants to pursue energy efficiency via intuitive thinking, prompting them to unconsciously change their power use habits to achieve the goal of energy conservation.

\section{Conclusions}

In this study, we examined the theory and methods concerning a HEAS, and employed a practical model to examine the feasibility of such a system. Recommendations and discussion are as follows:

(1) Complexity of power supply and demand

It is possible to control power supply and demand in the laboratory, and sequentially apply alternative energy, energy from storage, and mains electricity. However, load data for a real household is difficult to undergo batch processing. For instance, at times of peak consumption, when the household load is large, power may simultaneously come from alternative energy, storage batteries, and mains electricity.

(2) Wired or wireless transmission

Although the wireless transmission of power data is currently prevalent in the research world, since this study wishes to emphasize the investigation of the feasibility of a HEAS, it has initially adopted the high-stability transmission of signals through wires.

(3) Use of HEAS as an auxiliary element of HEMS

In this study, we designed a HEAS that can be read intuitively, in view of the fact that household members are typically very concerned about their electrical bills. However, although it cannot yet display power usage by household appliances and equipment, this system can be usefully employed as an auxiliary element in a HEMS. In this configuration, when users are aware of power usage, they will think about how they can change the use of household equipment in order to reduce their electrical charges.

\section{Acknowledgments}

This work was supported by MOST 103-2221-E-035-047 Building a Home Energy Aware System based on a smart grid infrastructure.

\section{References}

1 S. M. Kaplan and F. Sissine: Smart Grid (The Capitol.Net, New Taipei City, 2009) p. 124.

2 C. W. Gellings: The Smart Grid: Enabling Energy Efficiency and Demand Response (The Fairmont Press, Inc., 2009) p. 1.

3 T. Vijayapriya and D. P. Kothari: Smart Grid Renewable Energy 2 (2011) 305.

4 K. H. Yang: Building Energy Management (BEMS) handbook (Taiwan Green Productivity Foundation, Alexandria VA, 2008).

5 A. H. Mohsenian-Rad, V. W. S. Wong, J. Jatskevich, R. Schober, and A. Leon-Garcia: IEEE Trans. Smart Grid. 1 (2010) 320.

6 A. Khanna: Smart Grid Renewable Energy 3 (2012)165.

7 S. Sultan, U. Rana, I. Ahmed, and S. Rabbani: Smart Grid Renewable Energy 4 (2013)18.

8 Y. Z. Wang: HEMSs Development Analysis, Market Intelligence \& Consulting Institute, http://mic.iii.org.tw/ channelmic/channel_detail.asp?cno=199\&ccate=CHAN (accessed July 2015).

9 S. S. van Dam, C. A. Bakker, and J. C. Buiter: Energy Policy 63 (2013) 398.

10 R. Ford, J. Stephenson, N. Brown, and W. Stiehler: Energy Transitions: HEMSs (HEMS) (Centre for Sustainability, University of Otago, 2014). 
11 Y. Huang, H. Tian, and L. Wang: Int. J. Electr. Power Energy Syst. 73 (2015) 448.

12 NRGExpert: Barriers to the development of the smart grid (Global Smart Grid Report-Market Research, 2011) pp. 29-31.

13 Google: Google PowerMeter monitoring interface, www.google.com/powermeter/ (accessed March 2011).

14 Microsoft: Microsoft Hohm monitoring interface, www.microsoft-hohm.com (accessed March 2011).

15 San Der Technology: ASENs-910 series, smart meter and cloud real-time monitoring interface system, http:// www.a-sens.com/index.php?act=prod\&opl=43 (accessed May 2017).

16 iFamily: In-Snergy, http://ifamily.insnergy.com/html/intro2.jsp (accessed May 2017).

17 HITACHI: Energy Management System, http:/www.hitachi.com/businesses/infrastructure/product_site/cems/ management.html (accessed May 2017).

18 M. Weiser: The Computer of the 21st Century (Scientific American, 1991) p. 66.

19 B. Schilt, N. Adamsn, and R. Want: Context-Aware Computing Applications (The 1st International Workshop on Mobile Computing Systems and Applications, 1994) p. 85.

20 A. K. Dey and G. D. Abowd: Toward a better understanding of context and context-awareness. GVU Technical Report GIT-GVU-99-22, (College of Computing, Georgia Institute of Technology, 1999), ftp://ftp. cc.gatech.edu/pub/gvu/tr/1999/99-22.pdf (accessed May 2017).

21 J. M. Spool: What Makes a Design Seem 'Intuitive'?, UIE article, https://www.uie.com/articles/design intuitive/ (accessed May 2017).

22 B. J. Fogg: A behavior model for persuasive design, The 4th International Conference on Persuasive Technology (2009) Article No. 40.

23 M. G. Patterson: Energy Policy 24 (1996) 377.

\section{About the Authors}

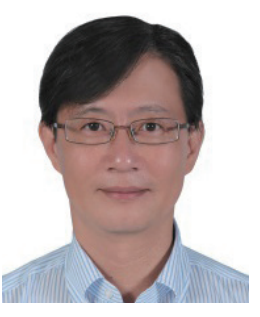

Shang Yuan Chen received his B.S. degree from Tunghai University, Taiwan, R.O.C., in 1992, M.S. from Yale University, USA, in 2000, and Ph.D. degree from National Cheng Kung University, Taiwan, R.O.C., in 2007. From 2008 to 2012, he was an assistant professor at Feng Chia University, Taiwan, R.O.C. Since 2012, he has been an associate professor at Feng Chia University. His research interests are in intelligent architecture and building information modeling.

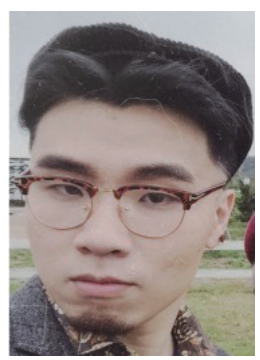

Zhen Lun Hu received his M.S. degree from Feng Chia University, Taiwan, R.O.C. His research interests are in MEMS, human-machine interface, and sensors. 